\title{
Gastrointestinal surgery in the COVID-19 pandemic
}

\author{
Marek Durlik \\ Clinical Department of Gastrointestinal Surgery and Transplantation, Centre of Postgraduate Medical Education, Warsaw, Poland \\ Gastroenterology Rev 2021; 16 (1): 1 \\ DOI: https://doi.org/10.5114/pg.2021.104732
}

\begin{abstract}
Address for correspondence: Marek Durlik MD, PhD, Clinical Department of Gastrointestinal Surgery and Transplantation, Centre of Postgraduate Medical Education, Warsaw, Poland, e-mail: mdurlik@post.home.pl
\end{abstract}

The data presented from a tertiary General University Hospital in Greece are interesting [1]. The comparable number of patients during the COVID-19 pandemic and the non-COVID era is a unique observation. A point to consider is that the study comes from a single centre, based on a relatively small number of cases.

The vast majority of other authors dealing with this topic have reported a significant decrease in the number of surgeries, regardless of whether they were qualified as urgent or elective.

A recently published multinational survey among WES (World Society for Emergency Surgery) members (18 collaborators from 31 countries) investigated the impact of the SARS-COVID-2 pandemic on emergency services and estimated the impact on emergency surgical patients. A decrease in the total number of patients undergoing emergency surgical procedures was reported by the majority $(87.8 \%)$ of surgeons. More than half of the responders observed more severe septic surgical emergencies, i.e. perforated appendicitis or necrotizing acute cholecystitis [2].

A decreased number of emergency surgical procedures and decreased number of surgical emergency patients during the COVID-19 pandemic was also reported by leading surgeons of non-university hospitals in Germany [3].

The reduction of available hospital beds and the operating room capacity were the principal factors of the abovementioned effect. Institutional changes, which entail human and logistic resources, were imposed in many European countries. The surgical beds increased the pool of ICU facilities but in the aftermath decreased the surgical activity.

Hubner et al. showed a $43 \%$ decrease of elective visceral procedures and a $25 \%$ decrease of emergency surgical procedures in a Swiss tertiary university hospital.

Moreover, they documented a $27 \%$ decrease in oncological surgeries [4].

Although postponing elective surgical procedures in the COVID-19 pandemic is universally accepted, the absence of treatment of resectable neoplastic tumours leads to progression of the disease and many complica- tions requiring emergency surgery. It is well documented that a 6-month delay in operation worsens the result by almost 30\% [5].

Analysing the data from hospitals from 190 countries, the CovidSurg Collaborative researchers estimated that nearly 30,000 operations were cancelled during the 12 -week period of the COVID-19 peak. Among these, $37 \%$ represented oncological cases [6].

In conclusion, the decrease of surgical activity in the COVID-19 pandemic should be restricted to elective surgery, i.e. hernia repair or cholecystectomy. Urgent surgical procedures and oncological surgery, especially in the case of low-risk resectable tumours, should be given special attention.

\section{Conflict of interest}

The author declares no conflict of interest.

\section{References}

1. Mulita F, Vailas M, Tchabashvili L, et al. The impact of the COVID-19 outbreak on emergency surgery: a Greek emergency department experience. Gastroenterology Rev 2021; 16 (1): 95.

2. Reichert M, Sartelli M, Weigand MA, et al. Impact of the SARSCoV-2 pandemic on emergency surgery services-a multinational survey among WSES members. World J Emerg Surg 2020; 15: 64 .

3. Stoß C, Steffani M, Kohlhaw K, et al. The COVID-19 pandemic: impact on surgical departments of non-university hospitals. BMC Surg 2020; 20: 313.

4. Hubner M, Zingg T, Nartin D, et al. Surgery for non Covid-19 patients during the pandemic. PLoS One https;/doi.org/10. 1371journal.pone.0241331 October 23,2020.

5. Sud A, Jones ME, Broggio J, et al. Collateral damage: the impact on outcomes from cancer surgery of the COVID-19 pandemic. Ann Oncol 2020; 31: 1065-74.

6. COVIDSurg Collaborative. Elective surgery cancellation due to the Covid-19 pandemic: global predictive modelling to inform surgical recovery plans. Br J Surg 2020; 107: 1440-9

Received: 15.03 .2021

Accepted: 15.03 .2021 